\title{
Selection of the Optimal Solvent Grade for the Mobile Phase in HPLC with Electrochemical Detection Based on FUMI Theory
}

\author{
Akira Kotani, ${ }^{* \dagger}$ Yanan YuAn, ${ }^{* * *}$ Bin YANG, ${ }^{* *}$ Yuzuru HaYaShI, $* * *$ Rieko Matsuda, ${ }^{* * *}$ and \\ Fumiyo KusU* \\ *School of Pharmacy, Tokyo University of Pharmacy and Life Sciences, \\ 1432-1 Horinouchi, Hachioji, Tokyo 192-0392, Japan \\ **Institute of Chinese Material Medica, China Academy of Chinese Medical Sciences, \\ 16 Dongzhimennei Nanxiaojie, Beijing 100700, China \\ ***National Institute of Health Sciences, 1-18-1 Kami-Yoga, Setagaya, Tokyo 158-8501, Japan
}

\begin{abstract}
The optimum conditions of the mobile phase for HPLC with electrochemical detection (HPLC-ECD) were selected from among solvents of different grades with the standard deviation (SD) of area measurements based on FUMI (function of mutual information) theory as a criterion. In HPLC-ECD for determining baicalin and baicalein using a phosphoric acid-methanol-water mixture (0.5:60:40, v/v/v) as a mobile phase, the effects of three grades of phosphoric acid, of five grades of methanol, and of seven grades of water on the measurement precision were examined. The mixture, if characterized by the minimum SD of measurements, was selected as the optimal mobile phase. This selection method saves considerable amounts of chemicals and experimental time, and would be a useful exploration technique for the routine check and troubleshooting of HPLC-ECD.
\end{abstract}

(Received March 3, 2009; Accepted May 21, 2009; Published July 10, 2009)

The optimization of a system of high-performance liquid chromatography with electrochemical detection (HPLC-ECD) is essential for the high-sensitive determination of redox compounds with extreme precision. Since the electron transfer process of the analyte depends on the molar flux of redox compounds, as well as the thermodynamics and kinetics of the oxidation or reduction, the size of the signal current is strongly affected by the column size, detector geometry, material of the working electrode, flow rate, composition of the mobile phase, and temperature. ${ }^{1-5}$ Our group has developed an optimization strategy using a chemometric tool, called FUMI (function of mutual information) theory, ${ }^{6}$ which provides a standard deviation (SD) and relative SD (RSD) of measurements from stochastic aspects of the signal and noise in a chromatogram without repeating the measurement of real samples. ${ }^{7}$

The elimination of chromatographic baseline noise, which consists of a non-faradic current and a faradic current, were found to be essential to increase the sensitivity of HPLC-ECD. ${ }^{7-10}$ The non-faradic current, which arises from charging the double layer at the interface between the working electrode and a solution of the mobile phase exists in any HPLC-ECD experiment. ${ }^{11,12}$ The faradic noise current results from not only unwanted redox reactions of trace impurities in a mobile phase, but also from an unwanted reaction of a working electrode surface. The non-faradic current noise is ubiquitous and not removable, but the faradic current noise may be removed by using highly pure solvents and chemicals in the mobile phase and by a proper selection of the applied potential where the working electrode is not oxidized or reduced.

† To whom correspondence should be addressed.

E-mail: kotani@toyaku.ac.jp
An evaluation method is desired for establishing a stable HPLC-ECD system with satisfactory precision, if the chromatographic baseline noise is reduced through manipulation of the mobile-phase components. The precision is generally described in terms of the SD of measurements. In well-known statistical ways, however, a large number of repetitions are required for obtaining an exact estimate of the measurement SD. ${ }^{13}$ To circumvent this problem, this paper adopts the FUMI theory.

In this study, we proved that the SD of area measurements based on FUMI theory is useful for selecting the optimal solvent and chemical grade to prepare the mobile phase in HPLC-ECD for determining baicalin and baicalein in Kampo medicines.

\section{Experimental}

\section{Reagents and chemicals}

Baicalin (>99\%), baicalein (>98\%), and propyl gallate (>99\%) were obtained from Wako Pure Chemical Industries, Ltd. (Osaka, Japan). The purity in the specification of JIS special, 1st grade, HPLC grade, and LC/MS grade methanol were $>99.8$, $>99.5$, >99.7, and $>99.7 \%$, respectively, and these were purchased from \#1 Co. LC/MS grade methanol (>99.7\%) was also purchased from \#2 Co. Ion exchange water (IEW), distilled water (DW), and ultrapure water were prepared in our laboratory by a commercial water distillation apparatus containing an ion-exchange resin column (\#3 Co.) and an ultrapure water system (\#4 Co.). HPLC and LC/MS grades of DW were purchased from \#1 and \#2 Co. The purities in the specification of JIS special, 1st grade, and HPLC grade phosphoric acid were $>85.0 \%$, which were purchased from \#1 Co. Because the 
present data would affect the benefit of companies, the names of the manufactures are not shown.

\section{Apparatus and HPLC-ECD conditions}

The HPLC-ECD equipment comprised a DP-2080-53 vacuum degasser (Jasco, Tokyo, Japan), a 301M pump (Flom, Tokyo, Japan), a 7725 injector fitted with a $5-\mu \mathrm{L}$ injection loop (Reodyne, Cotati, CA), a Capcell Pak C18 UG 120 microbore ODS column $(150 \mathrm{~mm} \times 1.0 \mathrm{~mm}$ i.d., $3 \mu \mathrm{m}$, Shiseido, Tokyo, Japan), an FT-1 column oven (BAS, Tokyo, Japan), and an LC-4C electrochemical detector (BAS). The electrochemical cell (Radial flow cell, BAS) was constructed from a glassy carbon working electrode, an $\mathrm{Ag} / \mathrm{AgCl}$ reference electrode, and a stainless-steel auxiliary electrode. A $5-\mu \mathrm{L}$ test solution or standard baicalin and baicalein solution was injected into a microbore ODS column maintained at $25^{\circ} \mathrm{C}$.

A degassed phosphoric acid-methanol-water mixture $(0.5: 60: 40, \mathrm{v} / \mathrm{v} / \mathrm{v})$ solution served as the mobile phase, at a flow rate of $30 \mu \mathrm{L} \mathrm{min}{ }^{-1}$. The detection potential for monitoring baicalin and baicalein was set at $+0.6 \mathrm{~V}$ vs. $\mathrm{Ag} / \mathrm{AgCl}$. An internal standard method was used for determining the baicalin and baicalein concentrations in the test solution, and propyl gallate was used as an internal standard (I.S.).

The analog data of a chromatogram from the electrochemical detector were converted to digital chromatogram data by a chromatographic workstation containing an A/D converter (ChemStation, Agilent Technology, Tokyo, Japan); the digital data were recorded by a personal computer at sampling intervals of $0.2 \mathrm{~s} \mathrm{point}^{-1}$. A calculation of the FUMI theory was carried out with commercial software, MAY2000 (Yazawa, Tochigi, Japan).

\section{Sho-saiko-to}

Granulated powder of Sho-saiko-to (EK-9) and liquid medicine of Sho-saiko-to (Cakonal) were purchased from Kurasie Co. (Tokyo, Japan) and Daiichi Sankyo Healthcare Co. (Tokyo, Japan), respectively.

A daily dose $(6.0 \mathrm{~g})$ of EK-9 and $(90 \mathrm{~mL})$ of Cakonal were extracted from $7.0 \mathrm{~g}$ Bupleuri radix, $5.0 \mathrm{~g}$ Pinelliae tuber, $3.0 \mathrm{~g}$ Scutellariae radix, $3.0 \mathrm{~g}$ Zizyphi fructus, $3.0 \mathrm{~g}$ Ginseng radix, $2.0 \mathrm{~g}$ Glycyrrhiza, and $1.0 \mathrm{~g}$ Zingiberis rhizoma.

\section{Preparation of test solution}

In order to prepare a test solution of Cakonal, Cakonal was diluted by $4 \%$ with water, and one part of this solution was diluted by adding 99 parts of a phosphoric acid-methanol-water mixture $(0.5: 60: 40, \mathrm{v} / \mathrm{v} / \mathrm{v})$. A $5-\mathrm{mL}$ volume of the diluted solution was added to $3.0 \mathrm{~mL}$ of $2.0 \mu \mathrm{g} \mathrm{mL}^{-1}$ I.S. solution, and diluted with a phosphoric acid-methanol-water mixture $(0.5: 60: 40, \mathrm{v} / \mathrm{v} / \mathrm{v})$ to $25 \mathrm{~mL}$. Moreover, in order to prepare a test solution of EK-9, EK-9 $(10 \mathrm{mg})$ was added to $10 \mathrm{~mL}$ of methanol, mixed for $1 \mathrm{~min}$, ultrasonicated for $30 \mathrm{~min}$, and centrifuged for $5 \mathrm{~min}$ at $3000 \mathrm{rpm}$. A $300-\mu \mathrm{L}$ volume of the supernatant was added to $3.0 \mathrm{~mL}$ of a $2.0 \mu \mathrm{g} \mathrm{mL}^{-1}$ I.S. solution, and diluted with a phosphoric acid-methanol-water mixture $(0.5: 60: 40, \mathrm{v} / \mathrm{v} / \mathrm{v})$ to $25 \mathrm{~mL}$. These test solutions were filtered through a membrane filter (pore size, $0.45 \mu \mathrm{m}$ ). Then, $5-\mu \mathrm{L}$ volumes of the test solutions were injected into the HPLC system.

\section{Theory}

In FUMI theory, attention is paid to the phenomenological properties of noise. A detailed explanation of FUMI theory to

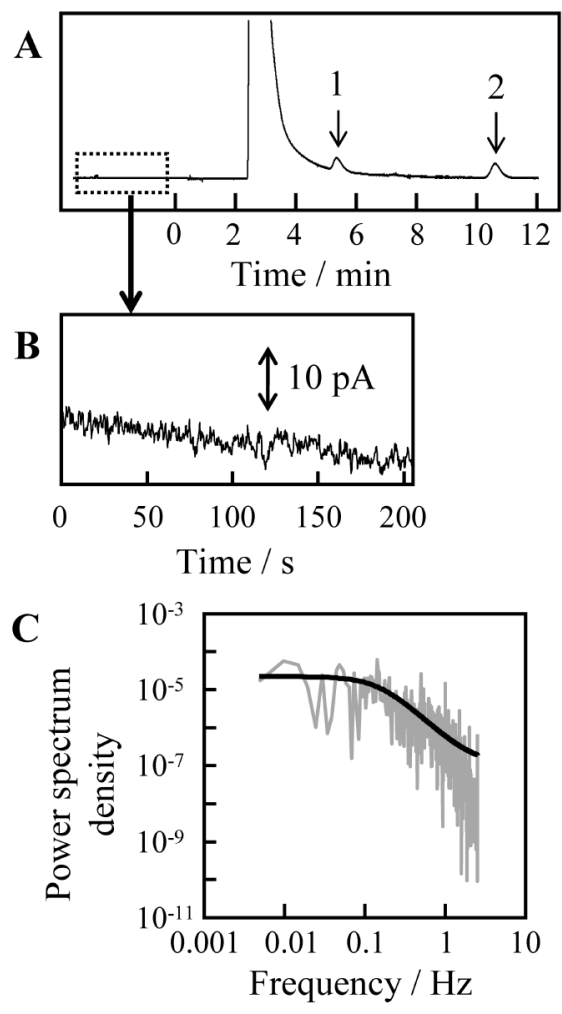

Fig. 1 (A) Chromatogram of $9.0 \mathrm{ng} \mathrm{mL}^{-1}$ baicalin and $1.4 \mathrm{ng} \mathrm{mL}^{-1}$ baicalein, (B) expanded chromatographic baseline noise, and (C) the power spectrum of chromatographic baseline noise in HPLC-ECD. (A) Peaks: 1, baicalin; 2, baicalein. The HPLC-ECD conditions were the same as described in the experimental section. (C) The digital data of 1024 data point $\left(0.2 \mathrm{~s}\right.$ point $\left.{ }^{-1}\right)$ converted from analog signals of chromatographic baseline were transferred to power spectra by Fourier transforms. The smooth solid line designates the best fit of the theoretical line (white noise + Markov process) to the observed power spectrum (zigzag line).

estimate the RSD of area measurements has already been given. ${ }^{6}$ If a chromatographic peak has a width of $k_{\mathrm{f}}$ data points and area, $A$, the $\mathrm{SD}(\sigma)$ of area measurements can be estimated based on the noise parameters of the $\mathrm{SD}, \tilde{w}$, of the white noise and the $\mathrm{SD}, \tilde{m}$, and correlation parameter, $\rho$, of the Markov process:

$$
\sigma^{2}=k_{\mathrm{f}} \tilde{w}^{2}+\frac{\tilde{m}^{2}}{(1-\rho)^{2}}\left(k_{\mathrm{f}}-2 \rho \frac{1-\rho^{\mathrm{kf}}}{1-\rho}+\rho^{2} \frac{1-\rho^{2 \mathrm{kf}}}{1-\rho^{2}}\right)
$$

Given a calibration line, the FUMI theory can provide a detection limit. Here, the detection limit, $L_{\mathrm{D}}$, is defined as $L_{\mathrm{D}}=$ $3 s / a$, where $s$ means the SD of the measurement ( $=\sigma$ of FUMI theory), and $a$ implies the slope of the calibration line. ${ }^{14}$ We can notice from the equation that $(s / a) / L_{\mathrm{D}}=1 / 3=33.3 \%$, the detection limit is the concentration, $L_{\mathrm{D}}$, at which the RSD of measurements, $(s / a) / L_{\mathrm{D}}$, shows $33.3 \%$. We should note that $s / a$ means the SD with the dimension of concentration, and thus $(s / a) / L_{\mathrm{D}}$ denotes the RSD with respect to the concentration.

\section{Results and Discussion}

SD and RSD of chromatographic measurements

A chromatogram of baicalin and baicalein obtained by the HPLC-ECD system is shown in Fig. 1A. The noise part of the 


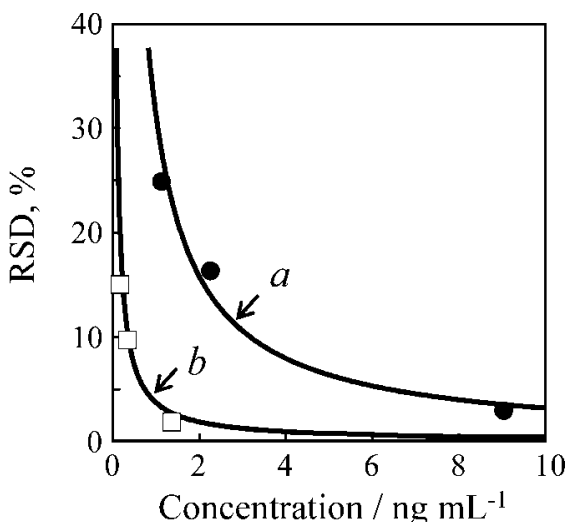

Fig. 2 Precision plots for the determination of baicalin and baicalein using HPLC-ECD. The observed RSDs calculated from chromatographic peaks of baicalin and baicalein are indicated by closed circles and open squares. The repetition number of measurements (n) was five at each concentration. The solid lines mean the predicted RSD based on FUMI theory using a chromatographic peak for $9.0 \mathrm{ng} \mathrm{mL}^{-1}$ baicalin (curve $a$ ) and $1.4 \mathrm{ng} \mathrm{mL}^{-1}$ baicalein (curve $b$ ), respectively. HPLC grade methanol from \#1 Co., ultrapure water, and JIS special grade phosphoric acid were used in this mobile phase.

chromatogram (Fig. 1B) was Fourier-transformed to the power spectra (Fig. 1C, zigzag line). As mentioned above, the noise parameters $(\tilde{w}, \tilde{m}$, and $\rho)$ were determined by a least-squares fitting of the model power spectrum (see the smooth line in Fig. 1C) to the real power spectrum. With the noise parameters and signal parameters (width and area), the SD and RSD of area measurements could be estimated according to FUMI theory. The goodness of fit of the least squares in Fig. 1C meets the prerequisite for a successful use of FUMI theory. That is, the badness of fit means that a random noise that cannot be treated by FUMI theory is included in the instrumental output, and it is impossible to apply the theory in this particular situation.

Figure 2 shows a plot of the measurement RSD against the sample concentration, called the precision profile. A discrete precision profile was obtained from five chromatograms each for $1.1,2.3$, and $9.0 \mathrm{ng} \mathrm{mL}^{-1}$ baicalin $(\bullet)$ and $0.17,0.34$, and $1.4 \mathrm{ng} \mathrm{mL}^{-1}$ baicalein $(\square)$. The continuous profile over a wide concentration range was drawn by FUMI theory using only one chromatogram for $9.0 \mathrm{ng} \mathrm{mL}^{-1}$ baicalin and $1.4 \mathrm{ng} \mathrm{mL}^{-1}$ baicalein (solid lines $a$ and $b$ in Fig. 2). The observed RSD $(n=5)$ values are in excellent agreement with the predicted RSD. This result illustrates the applicability of FUMI theory in the HPLC-ECD system to estimate the precision and detection limit without repeated measurements of real samples

With the continuous precision plot of Fig. 2, the detection limits of baicalin and baicalein were estimated to be 859 and $99 \mathrm{pg} \mathrm{mL} \mathrm{m}^{-1}$, respectively.

Selection of chemicals and solvents to prepare the mobile phase For the determination of baicalin and baicalein in the HPLC-ECD system with an ODS column and a mobile phase of a phosphoric acid-water-methanol mixture (0.5:60:40, v/v/v), the SD of area measurements was used as a criterion for selecting the optimum condition in a mobile phase from the commercially available chemicals and solvents of various grades. In the following experiments, unopened commercially available methanol, DW, and phosphoric acid were used to prepare the mobile phase in order to minimize the contamination. The SD of area measurements based on FUMI theory for five grades of
Table 1 SD of area measurements and detection limits of baicalin and baicalein by HPLC-ECD using a mobile phase of a phosphoric acid-methanol-water mixture (0.5:60:40, v/v/v)

A: Water (ultrapure water) and phosphoric acid (JIS special grade) were used

\begin{tabular}{|c|c|c|c|c|}
\hline \multirow{2}{*}{ Grade of methanol } & \multirow{2}{*}{ Manufacture } & \multirow{2}{*}{$\mathrm{SD} / \mathrm{pC}^{\mathrm{a}}$} & \multicolumn{2}{|c|}{$\begin{array}{l}\text { Detection limit/ } \\
\text { pg mL }\end{array}$} \\
\hline & & & Baicalin & Baicalein \\
\hline 1st grade & \#1 Co. & 15.10 & 409 & 55.4 \\
\hline JIS special grade & \#1 Co. & 11.92 & 322 & 42.5 \\
\hline HPLC grade & \#1 Co. & 12.58 & 464 & 65.1 \\
\hline LC/MS grade & \#1 Co. & 10.60 & 275 & 39.5 \\
\hline LC/MS grade & \#2 Co. & 13.82 & 329 & 43.4 \\
\hline
\end{tabular}

B: Methanol (LC/MS grade, \#1 Co.) and phosphoric acid (JIS special grade) were used

\begin{tabular}{|c|c|c|c|c|}
\hline \multirow[t]{2}{*}{ Grade of water } & \multirow[t]{2}{*}{ Manufacture } & \multirow[t]{2}{*}{$\mathrm{SD} / \mathrm{pC}^{\mathrm{a}}$} & \multicolumn{2}{|c|}{$\begin{array}{l}\text { Detection limit/ } \\
\text { pg mL } \mathrm{mL}^{-1}\end{array}$} \\
\hline & & & Baicalin & Baicalein \\
\hline IEW & Laboratory & 9.61 & 314 & 33.0 \\
\hline Ultrapure water & Laboratory & 9.60 & 197 & 28.4 \\
\hline DW & \#1 Co. & 9.78 & 231 & 30.6 \\
\hline DW HPLC grade & \#1 Co. & 6.86 & 213 & 26.6 \\
\hline DW HPLC grade & \#2 Co. & 8.46 & 335 & 38.9 \\
\hline DW LC/MS grade & \#1 Co. & 6.66 & 196 & 21.5 \\
\hline DW LC/MS grade & \#2 Co. & 9.14 & 290 & 31.2 \\
\hline
\end{tabular}

C: Methanol (LC/MS grade, \#1 Co.) and water (LC/MS grade, \#1 Co.) were used

\begin{tabular}{|c|c|c|c|c|}
\hline \multirow{2}{*}{$\begin{array}{c}\text { Grade of } \\
\text { phosphoric acid }\end{array}$} & \multirow{2}{*}{ Manufacture } & \multirow{2}{*}{$\mathrm{SD} / \mathrm{pC}^{\mathrm{a}}$} & \multicolumn{2}{|c|}{$\begin{array}{l}\text { Detection limit/ } \\
\text { pg mL } \mathrm{mL}^{-1}\end{array}$} \\
\hline & & & Baicalin & Baicalein \\
\hline 1st Grade & \#1 Co. & 9.00 & 306 & 30.7 \\
\hline JIS special grade & \#1 Co. & 6.66 & 196 & 21.5 \\
\hline HPLC grade & \#1 Co. & 6.86 & 206 & 22.1 \\
\hline
\end{tabular}

a. Pico coulomb $\left(\times 10^{-12}\right.$ coulomb): Chromatographic peak area in HPLC-ECD is expressed by electrical charge (= current $\times$ time). b. The water freshly prepared in our laboratory just before use.

methanol from two companies are given in Table 1A. In the certificate analysis of the acidity, alkalinity, ethanol concentration, and substance reducing permanganate for methanol, JIS grade was stringently tested compared with the 1st grade. HPLC grade methanol is guaranteed that the contents of substances that absorb UV light ranging from 210 to $400 \mathrm{~nm}$ are extremely low. Moreover, LC/MS-grade methanol having low content impurities have generally passed special quality-control tests to guarantee the purity, called suitability for LC/MS. The SD of area measurements using methanol of LC/MS grade is smaller than those using methanol of other grades. However, there was a little difference in the SD of area measurements between the LC/MS-grade methanol from \#1 Co. and \#2 Co.

HPLC grade DW is also guaranteed that the contents of substances that absorb UV light is extremely low, as well methanol. LC/MS-grade DW was examined concerning the total organic carbon (TOC), metal ion concentration, and/or suitability for LC/MS to guarantee the purity. As shown in Table 1B, the SD of area measurements for the LC/MS grade DW from \#1 Co. were the smallest among the water samples examined. Additionally, the SD of the area measurement was reduced using water freshly prepared in our laboratory just before use. 
In a certificate analysis for phosphoric acid, JIS special grade was examined concerning the limit of the impurity concentrations $\left(\mathrm{Cl}, \mathrm{SO}_{4}, \mathrm{Na}, \mathrm{K}, \mathrm{Cu}, \mathrm{Zn}, \mathrm{Cd}, \mathrm{Pb}, \mathrm{As}, \mathrm{Mn}, \mathrm{Fe}, \mathrm{Ni}\right.$, and substances reducing permanganate). However, because HPLC-grade phosphoric acid is controlled by the company's specification, the limit of the impurity concentration has not been made public, although the contents of substances that absorb UV light, ranging from 210 to $400 \mathrm{~nm}$, is extremely low, as guaranteed. In a comparison of the three grades of chemicals, phosphoric acid $(>85 \%)$, the SD of area measurements using JIS special grade showed the minimum SD, as can be seen in Table 1C. By the present strategy, the optimal solvent and chemical grade to prepare a mobile phase with less instrumental noise were selected.

The detection limits of baicalin and baicalein determination in our system are also given in Table 1. After the selection of methanol, water, and phosphoric acid to prepare the mobile phase, the detection limits were 196 and $21.5 \mathrm{pg} \mathrm{mL}^{-1}$ for baicalin and baicalein, respectively. The sampling intervals of an analog-to-digital converter in our system were $0.2 \mathrm{~s}$, and the acquisition time of the noise current of 1024 data points was no more than $3.5 \mathrm{~min}$. The power spectrum of the chromatographic baseline for only $3.5 \mathrm{~min}$ will also be useful for daily checks of the performance of an HPLC-ECD apparatus, as well as selecting the optimal solvent and chemical grade for HPLC-ECD. In this study, althought the ECD was used as an example in the development of the optimization strategy using the predicted SD of area measurements, the present strategy could be applied to HPLC systems with other detection units.

\section{Determination of baicalin and baicalein in Sho-saiko-to}

Baicalin and baicalein, which are the major ingredients of Scutellaria Root contained in a Kampo medicine called Sho-saiko-to, were determined by the present HPLC-ECD based on the internal-standard method. A chromatogram of baicalin and baicalein obtained from granulated powder of Sho-saiko-to (EK-9) is shown in Fig. 3.

The baicalin and baicalein contents in the Sho-saiko-to are listed along with their recovery data in Table 2. The RSD $(n=5)$ values in all cases were less than $2.44 \%$. The recovery of baicalin and baicalein from spiked test solutions was more than $97.1 \%$ and the RSD $(n=5)$ was less than $2.22 \%$, indicating that the present method provides quite accurate measurements of baicalin and baicalein in Sho-saiko-to. In addition, we found that baicalin was more abundant compared with baicalein in the Sho-saiko-to studied. Thus, the applicability of the present HPLC-ECD to determine baicalin and baicalein in Sho-saiko-to has been demonstrated.

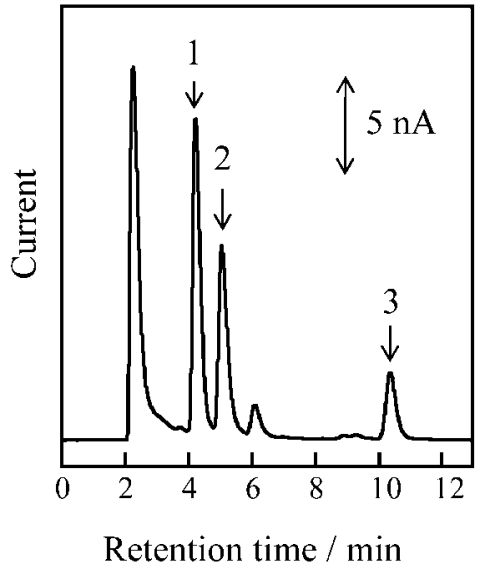

Fig. 3 Chromatogram of baicalin and baicalein obtained from granulated powder of Sho-saiko-to (EK-9). Peaks: 1, propyl gallate (I.S.); 2, baicalin; 3, baicalein. The HPLC-ECD conditions were the same as described in the experimental section.

\section{Conclusion}

An optimization strategy using an SD estimate of measurements based on FUMI theory has been applied to selecting the optimal solvent and chemical grade for preparing the mobile phese in HPLC-ECD. The time required for acquiring the prerequisite of FUMI theory (power spectrum) was sufficiently short for practical use.

It is common in HPLC that improvement, selection, and routine checks are carried out based on the trial-and-error of experienced operators. The methodology of the present paper should provide a new direction for routine checks and opitimizations of HPLC-ECD by saving not only considerable amounts of chemicals and energy, but also time and effort.

\section{Acknowledgements}

This work was supported by a grant from an International S\&T Cooperation Program from the Ministry of Science and Technology of the P. R. China (Grant No. 2006DFB31720).

Table 2 Contents of baicalin and baicalein in Sho-saiko-to and recovery from Sho-saiko-to spiked with baicalin and baicalein standards by HPLC-ECD

\begin{tabular}{|c|c|c|c|c|c|c|}
\hline \multirow{2}{*}{$\begin{array}{l}\text { Sample name } \\
\text { (dosage form) }\end{array}$} & & \multicolumn{2}{|c|}{ Content $(n=5)$} & \multicolumn{3}{|c|}{ Recovery $(n=5)$} \\
\hline & & Amount & RSD, $\%$ & Added amount & Recovery, $\%$ & RSD, $\%$ \\
\hline Cakonal & Baicalin & $3.56 \mathrm{mg} \mathrm{mL}^{-1}$ & 0.54 & $4.00 \mathrm{mg} \mathrm{mL}^{-1}$ & 97.1 & 0.68 \\
\hline (liquid medicine) & Baicalein & $0.1 \mathrm{mg} \mathrm{mL}^{-1}$ & 1.49 & $0.1 \mathrm{mg} \mathrm{mL}^{-1}$ & 104.3 & 2.22 \\
\hline EK-9 & Baicalin & $28.8 \mathrm{mg} \mathrm{g}^{-1}$ & 1.25 & $30.0 \mathrm{mg} \mathrm{g}^{-1}$ & 103.3 & 1.88 \\
\hline (granulated powder) & Baicalein & $1.34 \mathrm{mg} \mathrm{g}^{-1}$ & 2.44 & $1.30 \mathrm{mg} \mathrm{g}^{-1}$ & 97.4 & 1.97 \\
\hline
\end{tabular}

For the recovery test, baicalin and baicalein standards at each amount were directly spiked to each Sho-saiko-to. Baicalin and baicalein derived from both Sho-saiko-to and the standard were extracted, and a test solution was injected into HPLC-ECD to obtain a chromatogram. The recovery was $100 \%$ when the baicalin or baicalein amount in the recovery test was equal to the sum of baicalin or baicalein in Sho-saiko-to and the spiked standard. 


\section{References}

1. A. J. Bard and L. R. Faulkner, "Electrochemical Methods, Fundamentals and Applications", 1980, John Wiley and Sons, Inc., New York.

2. C. E. Lunte, P. T. Kissinger, and R. E. Shoup, Anal. Chem., 1985, 57, 1541.

3. R. D. Rocklin, T. R. Tullsen, and M. G. Marucco, $J$. Chromatogr., A, 1994, 671, 109.

4. J. M. Elbicki, D. M. Morgan, and S. G. Weber, Anal. Chem., 1984, 56, 978.

5. P. T. Kissinger, in "Encyclopedia of Chromatography", ed. J. Cazes, 2nd ed., 2005, Taylor \& Francis, FL, 517.

6. Y. Hayashi and R. Matsuda, Anal. Chem., 1994, 66, 2874.

7. A. Kotani, Y. Hayashi, R. Matsuda, and F. Kusu, J. Chromatogr., A, 2003, 986, 239.
8. H. W. Van Rooijen and H. Poppe, J. Liq. Chromatogr., 1983, 6, 2231.

9. J. A. Helpern, J. R. Ewing, and K. M. A. Welch, J. Chromatogr., 1982, 240, 491.

10. G. Blo, M. Remelli, F. Pedrielli, L. Balconi, F. Sigon, and F. Dondi, J. Chromatogr., 1991, 556, 249.

11. K. Xu, M. S. Ding, and T. R. Jow, Electrochim. Acta, 2001, $46,1823$.

12. I. N. Acworth, M. Naoi, H. Parvez, and S. Parvez, "Coulometric Electrode Array Detectors for HPLC", Progress in HPLC-HPCE, 1997, Vol. 6, VSP, Utrecht, The Netherlands.

13. J. C. Miller and J. N. Miller, "Statistics for Analytical Chemistry", 1988, Eills Horwood, West Sussex, UK.

14. G. L. Long and J. D. Winefordner, Anal. Chem., 1983, 55, $712 \mathrm{~A}$. 